\title{
RESPOSTAS NEUROMUSCULARES AO TREINAMENTO RESISTIDO DE ALTA INTENSIDADE (HIRT)
}

\author{
Antenor Barbosa Calandrini Azevedo \\ Universidade Federal do Pará, Belém, Pará, Brasil \\ Deivison Soares Meninea \\ Universidade Federal do Pará, Belém, Pará, Brasil \\ Arthur Pinto Magno \\ Universidade Federal do Pará, Belém, Pará, Brasil \\ Victor Silveira Coswig \\ Universidade Federal do Pará, Belém, Pará, Brasil
}

\begin{abstract}
Resumo
O presente estudo buscou avaliar respostas neuromusculares ao treinamento resistido de alta intensidade (HIRT). Doze universitários do sexo masculino com idade de $21 \pm 3$ anos, realizaram o protocolo HIRT, que é caracterizado por pausas de 20 segundos entre as repetições. Posteriormente, foi realizado salto vertical (SV), horizontal (SH), arremesso de Medicine-Ball (MedBall) e força de pressão manual (FPM), antes, depois e após 24h e 48h, o HIRT. Os saltos apresentaram significância nos períodos PRÉ e PÓS-SV $(p=0,01)$, SH $(p=0,02)$. Entretanto, arremesso de MedBall e FPM não demonstraram diferenças significativas. Especula-se que o HIRT afete de modo agudo a potência de membros inferiores, mas não a de superiores. Porém, o período de 24 horas parece ser suficiente para a recuperação.

Palavras-chave: Treinamento de resistência. Recuperação. Intensidade de treinamento. Educação física e treinamento.

\section{NEUROMUSCULAR RESPONSES TO HIGH INTENSITY RESISTANCE TRAIN- ING (HIRT)}

\begin{abstract}
The present study aimed to evaluate neuromuscular responses to high intensity resistance training (HIRT). Twelve male university students aged $21 \pm 3$ years, in which they performed the HIRT protocol, which is characterized by pauses of $20 \mathrm{sec}$ between repetitions. Subsequently, horizontal (SH) vertical jumps (SV), Medicine Ball (MedBall) and manual pressure force (FPM) were performed before, after and after $24 \mathrm{~h}$ and $48 \mathrm{~h}$ the HIRT. The jumps showed significance in the periods PRÉ and POS SV $(p=0.01), S H(p=0.02)$. However, MedBall and FPM throw did not show significant difference. HIRT acutely affects the potency of lower limbs, but not of upper limbs. However, the 24-hour period seems to be sufficient for recovery.
\end{abstract}

Keywords: Resistance training. Recovery. Training intensity. Physical education and training. 


\section{RESPUESTAS NEUROMUSCULARES AL ENTRENAMIENTO DE RESISTENCIA DE ALTA INTENSIDAD (HIRT)}

\section{Resumen}

El presente estudio buscó evaluar respuestas neuromusculares al entrenamiento resistido de alta intensidad (HIRT). Doce universitarios varones con edad de $21 \pm 3$ años, en el que realizaron el protocolo HIRT, que se caracteriza por pausas de 20 seg entre repeticiones. Se realizaron saltos verticales (SV) horizontales (SH), lanzamiento de Medicina-Ball (MedBall) y fuerza de presión manual (FPM), antes, después y después de $24 \mathrm{~h}$ y $48 \mathrm{~h}$ el HIRT. Los saltos presentaron significancia en los períodos PRÉ y PÓS SV $(p=0,01)$, SH $(p=0,02)$. Sin embargo, el lanzamiento de MedBall y FPM no mostró diferencia significativa. El HCA afecta de forma aguda a la potencia de miembros inferiores, pero no de superiores. Sin embargo, el período de $24 \mathrm{~h}$ parece suficiente para la recuperación.

Palabras-clave: Entrenamiento de resistencia. Recuperación. Intensidad de entrenamiento.

Educación física y entrenamiento.

\section{Introdução}

O treinamento resistido caracteriza-se por ações musculares contra a resistência de oposição, utilizando-se de pesos, bandas elásticas e/ou exercícios pliométricos (KRAEMER; RATAMESS, 2004), que envolvem as manifestações da força como resistência muscular localizada, hipertrofia, força máxima e potência (ACSM,2009). De modo geral, a modalidade apresenta elevado número de praticantes que buscam benefícios relacionados ao sistema cardiovascular, endócrino e densidade mineral óssea (STONE; PLISK; COLLINS, 2002).

Dentre as possíveis variações no treinamento resistido, protocolos de alta intensidade (AI) envolvem estímulos de curta duração e pausas curtas que promovem exaustão e, consequentemente, permitem redução da frequência semanal (MORO et al., 2017). Esse modelo se mostra interessante quando o objetivo envolve eficiência temporal, visto que possibilita a redução do volume das sessões (SABIDO; PEÑARANDA; HERNÁNDEZ-DAVÓ JL, 2016), o que pode ser uma alternativa para aumentar a adesão de pessoas que indicam a falta de tempo como barreira para a prática de programas de treinamento resistido e contrapõe os modelos vigentes baseados em volumes elevados (LIZ, DE E ANDRADE, 2016).

Considerando as respostas agudas neuromusculares em relação ao treinamento de AI, em um estudo com 14 sujeitos, Marshall et al. (2018) evidenciaram que o método de pausa e descanso promoveu maior recrutamento muscular e na taxa de desenvolvimento de força por meio de eletromiografia. Ainda Weakley et al. (2017) verificaram respostas neuromusculares por meio do countermovement jump após dois treinamentos de AI distintos, super-set e tri-set respectivamente, as medidas foram coletadas imediatamente e 24 horas após os protocolos. Os resultados demonstraram queda do desempenho de salto para ambos os treinamentos, que se prolongou até o período de $24 \mathrm{~h}$.

Entretanto, exercícios praticados em AI com períodos insuficientes de recuperação, como em dias consecutivos, promovem elevação relevante de radicais livres, o que pode aumentar os riscos de diversas doenças, como enfisema pulmonar, doenças inflamatórias, aterosclerose, câncer, além do envelhecimento precoce (EBBELING; CLARKSON, 1989). No entanto, existe um número reduzido de evidências do treinamento AI com pausa e descanso em relação ao sistema neuromuscular de maneira aguda. Portanto, torna-se necessário aperfeiçoar a prescrição do treinamento resistido através da relação de períodos de treinamentos com AI e o tempo de recuperação do sistema neuromuscular, evitando desgastes excessivos. Neste contexto, o objetivo do presente estudo foi avaliar as respostas neuromusculares ao protocolo HIRT 


\section{Materiais e Métodos}

\section{Participantes}

Participaram do estudo 12 universitários do sexo masculino, idade $21 \pm 3$ anos, massa corporal $76,1 \pm 12,1 \mathrm{~kg}$ e estatura $1,72 \pm 0,14 \mathrm{~m}$. Como critério de inclusão, foram considerados: possuir experiência mínima no treinamento resistido (TR) de três meses, já ter praticado os exercícios que foram utilizados na pesquisa, não possuir problemas osteomioarticulares, fatores de riscos como hipertensão e diabetes. Para os critérios de exclusão, foram estabelecidos: a frequência dos indivíduos em todos os dias de avaliações, não realizarem qualquer outro método de treinamento durante as coletas e desconfortos articulares durante a bateria de testes.

\section{Variáveis}

Para o presente estudo foram consideradas as variáveis independentes: o desempenho nos testes de seis repetições máximas (6RM), salto horizontal (SH), salto vertical (SV), arremesso de Medicine-Ball (MedBall), força de pressão manual (FPM), questionário de humor, escala subjetiva de percepção de esforço, escala de prontidão. Já como variáveis dependentes, foram consideradas: potência de membros inferiores e superiores, o intervalo específico para recuperação do sistema neuromuscular.

\section{Delineamento}

Inicialmente os participantes leram e assinaram o Termo de Consentimento Livre e Esclarecido, conforme a resolução no 466/12 do Conselho Nacional de Saúde do Brasil para pesquisas com seres humanos. Posteriormente, os indivíduos passaram pela anamnese através do questionário MIR-Q (CLARSEN; MYKLEBUST; BAHR, 2013) e foram devidamente informados sobre os procedimentos da pesquisa. As coletas ocorreram em seis encontros, no primeiro e segundo encontro os participantes foram submetidos a teste e reteste de 6RM, de modo a quantificar a carga ideal para a realização do HIRT. No terceiro encontro foi realizada a aferição e aplicação da escala de BRUMS (LAN et al., 2012). Em seguida foram executados os testes de SH, SV, arremesso de MedBall e FPM. Logo após a realização desses testes, os participantes foram submetidos ao protocolo HIRT. Posteriormente, os indivíduos retornaram a fazer as avaliações pós-protocolo e nos períodos de $24 \mathrm{~h}$ e $48 \mathrm{~h}$.

\section{Procedimentos e Medidas Avaliadas}

\section{Escala de prontidão}

O questionário utilizado para a escala de prontidão foi realizado através do MIR-Q, tendo como características ser fechado e visar a anamnese dos candidatos. O questionário possui perguntas sobre lesões musculoesqueléticas, dor, instabilidade, bloqueio articular, edema, desvio na coluna vertebral e desgaste excessivo nas articulações (CLARSEN; MYKLEBUST; BAHR, 2013).

\section{Teste de 6RM}

Para mensurar a carga do HIRT em 6RM, foram utilizados o Supino e o Leg 45. O 
protocolo se inicia com $40 \%$ de uma carga equivalente a 6RM para realização do aquecimento, contendo 2 séries de 15 repetições com 2 minutos de pausa entre cada sessão, consequentemente, os testes se iniciam com a carga total, caso o participante consiga fazer 6RM o teste é paralisado imediatamente. Se o indivíduo conseguir realizar acima de 6RM, passam a ser acrescentados de $2 \%$ a $10 \%$ sobre a mesma carga, entretanto, se as repetições forem abaixo de $6 \mathrm{RM}$, retira-se a mesma porcentagem de carga. O protocolo teve o total de cinco tentativas para estabelecer o peso exato dos 6RM, o tempo de pausa em cada série foi estabelecido em 3 minutos, e 7 minutos entre os exercícios. Após $48 \mathrm{~h}$ realizou-se o reteste utilizando a carga adquirida no primeiro teste, repetindo assim os mesmos procedimentos (MIDDLETON; YAFFE, 2009).

\section{Desempenho em saltos}

Para as avaliações das medidas de potência de membros inferiores utilizou-se o SV no qual se realizou primeiramente a demarcação do ponto de referência pela extremidade distal dos dedos da mão dominante. Consequentemente, foram realizadas as medidas de cada salto através de uma fita métrica na vertical. Cada participante posicionou-se com os joelhos flexionados com as mãos voltadas para trás, gerando a propulsão máxima, para tocarem com as pontas dos dedos no ponto mais alto, cada indivíduo realizou três tentativas com o objetivo de avaliar o salto mais alto e a média. $\mathrm{O}$ cálculo de cada salto foi realizado pela subtração do ponto de referência, em relação à medida de cada salto (CASTRO-PINERO et al., 2009). Além disso, foi feito o $\mathrm{SH}$, sendo realizadas também três tentativas para obter o salto com a maior distância possível. Os voluntários ficaram atrás de uma faixa sem ultrapassá-la, em seguida foram auxiliados para manter o espaçamento entre os pés da largura dos ombros, na posição de agachamento para gerar maior propulsão. Sendo permitidos os movimentos dos braços durante o salto para proporcionar uma melhor estabilização durante a aterrissagem. A forma de medir a distância de cada salto foi através de uma fita métrica colocada na vertical. Foram validados somente os saltos nos quais os calcanhares do participante estiveram completamente fixados ao chão, caso contrário, o teste foi anulado (CASTRO-PIÑERO et al., 2010).

\section{Arremesso de MedBall}

Já para potência dos membros superiores foi realizado o MedBall, sendo que os participantes posicionaram-se com as costas perpendicularmente à parede, a articulação do quadril formando o ângulo de $90^{\circ}$, joelhos completamente estendidos e unidos e os cotovelos flexionados. O MedBall (Performance, Musa, Brasil), pesando $3 \mathrm{~kg}$, foi devidamente direcionado ao peitoral dos indivíduos, sendo que eles foram orientados a arremessar a MedBall com maior força possível. A demarcação foi realizada por uma fita métrica do ponto de saída do arremesso até a maior distância possível, foram realizadas no total três tentativas. O MedBall foi banhado com cal para demarcar a posição específica na qual a bola toca ao solo (FETT et al., 2018).

\section{Força de Preensão Manual}

Posteriormente, foi determinada a FPM através da dinamometria isocinética (Dinamômetro Manual capacidade de $50 \mathrm{kgf}$, Crow, Brasil) que mede a força aplicada por uma pessoa sentido de fechamento da mão por meio de contrações isométricas. Os indivíduos permaneceram confortavelmente sentados, ombros abduzidos, a articulação do cotovelo formando um ângulo de $90^{\circ}$, punho na posição neutra. A mensuração da força é avaliada através 
de três tentativas, prevalecendo a tentativa que possuir maior grau de amplitude de força, com intervalos de 15 segundos, para evitar a fadiga. A estimativa do tempo de contração foi de 3 segundos, com o intuito de não ocasionar alterações bruscas na frequência cardíaca e pressão arterial (PFISTER et al., 2018).

\section{Escala de BRUMS}

Em relação às variáveis psicológicas, foi utilizado o questionário BRUMS adaptado do "Profile of Mood States", no qual possui indicadores simples de humor (raiva, tensão, depressão, fadiga, confusão mental e vigor), cada variável psicológica possui uma escala de 5 pontos, $(0=$ nada, 4 = extremamente $)$. A pergunta foi elaborada da seguinte forma: "como você se sente agora", "como você se sentiu nesta última semana, inclusive hoje?" A escala de BRUMS foi realizada em dois momentos: pré e logo após o protocolo HIRT (LAN et al., 2012).

\section{Protocolo HIRT}

O protocolo foi previamente adaptado, possuindo um número menor de exercícios em relação ao método original. Portanto, estabeleceu-se em três momentos, primeiramente são realizadas seis repetições, seguidas de pausas de 20 segundos, no segundo momento foram realizados 2/3RM, com 20 segundos de pausa, no terceiro momento $2 / 3 \mathrm{RM}$, com 2'30" de pausa entre as séries, estabelecendo o primeiro conjunto no total de 3 . A porcentagem de carga para realização do HIRT varia de $75 \%$ a $85 \%$ de 6RM, a cadência do movimento foi de 2 segundos na fase concêntrica e 2 segundos de fase excêntrica (PAOLI et al., 2012).

Os exercícios utilizados para o HIRT foram, primeiramente, o supino realizado na posição em decúbito dorsal e os cotovelos parcialmente estendidos, utilizando-se uma pegada na barra com o dorso das mãos voltadas para cima e com afastamento igual à distância dos acrômios, joelhos e quadris semiflexionados, com os pés sobre o apoio do próprio aparelho, a partir da fase excêntrica $\left(90^{\circ}\right.$ entre braço e antebraço) realizou-se a extensão completa dos cotovelos e flexão horizontal dos ombros (MIRANDA et al., 2005). Logo após realizou-se o leg press $45^{\circ}$ que se estabeleceu da seguinte maneira: cada participante foi orientado a sentarse no banco e posicionar os pés na plataforma, com o espaçamento na largura da distância dos acrômios, segurando os pegadores laterais, destravando o aparelho e abaixando a carga de maneira lenta, até o ponto no qual os joelhos formem um ângulo de $90^{\circ}$, consequentemente, o participante empurra a plataforma a partir dos calcanhares e estendendo os joelhos sem movimentos bruscos, estacionando o movimento antes que a articulação do joelho esteja completamente estendida (SAKUGAWA et al., 2018). Por fim, a sessão de treinamento durou aproximadamente 19 minutos, incluindo o período de aquecimento.

\section{Escala subjetiva de BORG}

A escala subjetiva de $B O R G$ teve como princípio medir a percepção de esforço dos indivíduos após o método de treinamento aplicado. Com a escala de 0 a 10 , numeração de acordo com a percepção de esforço dos participantes, determinada pelos diferentes níveis de dificuldade que variam entre: muito fácil ao exaustivo. Após a prática do treinamento os participantes receberam uma ficha, na qual está indicada a sua dificuldade no método (BORG, 1982). 


\section{Análise estatística}

Após aplicação do teste de Shapiro-Wilk para verificação da normalidade dos dados, eles são apresentados por meio de média, como medida de centralidade, e desvio padrão como medida de dispersão. Para as comparações das médias referentes aos testes de desempenho físico entre momentos, foi aplicada Análise de Variância (ANOVA) para medidas repetidas e, quando adequado, as diferenças foram testadas por meio do post-hoc de Bonferroni. Já para as comparações entre momentos das variáveis do teste de BRUMS foi aplicado teste $t$ de student para amostras pareadas. Todos os testes foram executados no software SPSS versão 22.0 e para todas as análises foi considerada significância estatística quando $p \leq 0,05$.

\section{Resultados}

Os resultados de força e potência são apresentados na Tabela 1. Nela percebe-se que, quanto à potência de membros inferiores, a análise de medidas repetidas indicou diferença significante para desempenho em SV $\left(F=3,83 ; p=0,021 ; \eta^{2}=0,29\right)$ e $\mathrm{SH}(\mathrm{F}=7,58 ; \mathrm{p}=0,001$; $\left.\eta^{2}=0,45\right)$. Porém, a análise de post-hoc sinalizou significância apenas entre os momentos PRÉ e PÓS, tanto para $\mathrm{SV}(\mathrm{p}=0,01)$ quanto para $\mathrm{SH}(\mathrm{p}=0,02)$. Já quanto a potência e força de membros superiores, no teste de arremesso de MedBall não foram identificadas diferenças significativas $\left(F=1,586 ; p=0,216 ; \eta^{2}=0,150\right)$, bem como quanto a FPMD $(F=0,734 ; p=$ 0,$\left.541 ; \eta^{2}=0,075\right)$ e FPME $\left(F=0,735 ; p=0,540 ; \eta^{2}=0,075\right)$.

Tabela 1 - Média, valores máximos, mínimos, variação e desvio padrão dos testes de potência de membros inferiores SV, SH, MedBall e FPM de ambas as mãos

\begin{tabular}{ccccc}
\hline & MÉDIA \pm DP & MÍNIMO & MÁXIMO & CV(\%) \\
\hline Salto vertical (cm) & & & & \\
PRÉ & $53,0 \pm 8,33$ & 38,00 & 76,00 & 15,00 \\
PÓS & $50,0 \pm 7,37 *$ & 56,00 & 64,00 & 14,70 \\
24h & $52,1 \pm 6,95$ & 60,00 & 62,00 & 13,30 \\
$48 \mathrm{~h}$ & $52,0 \pm 7,91$ & 60,00 & 66,00 & 15,20 \\
Salto Horizontal & & & & \\
(m) & & & & 7,76 \\
PRÉ & $2,19 \pm 0,17$ & 1,92 & 2,58 & 6,96 \\
PÓS & $2,01 \pm 0,14 *$ & 1,80 & 2,27 & 7,51 \\
24h & $2,13 \pm 0,16$ & 1,84 & 2,54 & 9,25 \\
$48 \mathrm{~h}$ & $2,16 \pm 0,20$ & 1,78 & 2,57 & \\
MebBall (m) & & & & 10,30 \\
PRÉ & $4,65 \pm 0,48$ & 4,01 & 5,53 & 8,80 \\
PÓS & $4,43 \pm 0,39$ & 3,60 & 4,99 & \\
\hline
\end{tabular}




\begin{tabular}{ccccc}
\hline 24h & $4,65 \pm 0,37$ & 4,15 & 5,34 & 7,95 \\
$48 \mathrm{~h}$ & $4,56 \pm 0,34$ & 4,01 & 5,31 & 7,45 \\
FPMD (kgf) & & & & \\
PRÉ & $43,1 \pm 6,04$ & 34,50 & 50,00 & 14,00 \\
PÓS & $43,8 \pm 5,40$ & 37,50 & 50,00 & 12,30 \\
$24 \mathrm{~h}$ & $41,5 \pm 6,16$ & 32,00 & 50,00 & 14,80 \\
$48 \mathrm{~h}$ & $42,9 \pm 4,80$ & 37,50 & 50,00 & 11,10 \\
FPME (kgf) & & & & \\
PRÉ & $41,3 \pm 5,74$ & 34,50 & 50,00 & 13,80 \\
PÓS & $41,2 \pm 6,08$ & 30,50 & 50,00 & 14,70 \\
$24 \mathrm{~h}$ & $40,2 \pm 6,11$ & 33,00 & 50,00 & 15,10 \\
$48 \mathrm{~h}$ & $40,8 \pm 5,07$ & 35,00 & 50,00 & 12,40
\end{tabular}

Legenda: FPMD-força de pressão manual braço direito. FPME-força de pressão manual braço esquerdo. DP-desvio padrão. CV-coeficiente de variação em porcentagem. MebBallmedicine-ball.* Estatisticamente diferente do valor PRÉ.

Quanto às respostas psicométricas, a Tabela 2 apresenta as pontuações decorrentes da escala de BRUMS. Nela percebe-se diferença significativa em relação ao estado de fadiga $(\mathrm{p}=0,005)$, vigor $(\mathrm{p}=0,026)$ e tensão $(\mathrm{p}=0,018)$. Adicionalmente, destaca-se que a média da escala de BORG obtida após a sessão de HIRT foi de $8,9 \pm 0,5$.

Tabela 2 - Média e desvio padrão dos valores obtidos pela escala de BRUMS

\begin{tabular}{lllcr}
\hline \multicolumn{4}{l}{ PRÉ } & \multicolumn{1}{l}{ PÓS } \\
\hline MÉDIA \pm DP & MÉDIA \pm DP & Z & p-valor \\
\hline Fadiga & $0,08 \pm 0,27$ & $0,16 \pm 0,55$ & $-0,447$ & 0,655 \\
Confusão & $1,00 \pm 0,81$ & $3,75 \pm 0,43$ & $-2,821$ & 0,005 \\
Raiva & $0,41 \pm 0,49$ & $1,00 \pm 0,91$ & $-1,725$ & 0,084 \\
Vigor & $0,66 \pm 0,94$ & $1,16 \pm 1,57$ & $-0,750$ & 0,453 \\
Tensão & $2,91 \pm 0,49$ & $1,83 \pm 1,21$ & $-2,232$ & 0,026 \\
\hline
\end{tabular}

Legenda: DP- Desvio padrão

\section{Discussão}

O objetivo deste estudo foi avaliar respostas neuromusculares ao protocolo HIRT. Dentre os principais achados, indica-se que a execução do protocolo promoveu impacto relevante, de modo agudo, na potência de membros inferiores. Porém, os valores retornaram aos 
níveis de base após $24 \mathrm{~h}$ de recuperação. Já o desempenho de força e potência de membros superiores não foi afetado pelo protocolo.

Considerando a hipótese de queda do desempenho e tempo de recuperação de potência de membros inferiores, o estudo de Freitas et al. (2014) verificou efeito deletério na altura de saltos com contramovimento após jogos consecutivos de futsal. Os resultados indicaram queda de pré $(38,6 \pm 3,5 \mathrm{~cm})$ para $24 \mathrm{~h}$ após o jogo $1(37,7 \pm 3,3 \mathrm{~cm})$ e para $24 \mathrm{~h}$ após jogo $2(36,9$ $\pm 3,6 \mathrm{~cm}$ ). Todavia, o estudo de Lopes (2013) realizou duas sessões de exercício de força com intervalo de recuperação de $24 \mathrm{~h}$ ou $48 \mathrm{~h}$. O protocolo consistia nos exercícios de supino inclinado e declinado executados em 5 séries de 10 repetições com intensidade de $70 \%$ de $1 \mathrm{RM}$ para cada exercício. Os resultados demonstraram que ambas as sessões ( $24 \mathrm{~h}$ e $48 \mathrm{~h}$ ) apresentaram redução significante $(\mathrm{p}<0,05)$ na capacidade neuromuscular (força, potência e velocidade), porém, 24h após o treinamento houve retorno aos valores PRÉ ( $p>0,05)$. Desse modo, sugere-se que os saltos induziram seletivamente à fadiga periférica, mas os mecanismos centrais parecem não terem sido afetados a ponto de promover a perda aguda de força e potência (CLEBIS; RAQUEL; NATALI, 2001).

Nesse sentido, sugere-se que marcadores bioquímicos se elevam durante exercícios de AI, com essa perspectiva, o estudo de Weakley et al. (2017) analisou o grau de lesão na musculatura através de exercícios de AI em jogadores universitários de Rúgbi. Os resultados demonstraram elevação da concentração de creatina kinase (CK) em 24h. Em contrapartida, tais aspectos não seriam responsáveis por prejudicar o desempenho de potência, visto que essa é uma capacidade física que requer velocidade de condução por meio das fibras musculares e modificações do número de sinapses das unidades motoras (HUNTER et al., 2001).

Em relação à FPM, não houve diferença significativa em nenhum momento das análises, contudo, o estudo de Baptista et al. (2013) verificou o torque de PM com cargas submáximas de contração voluntária isométrica máxima, entre $20 \%, 40 \%$ e $60 \%$. Os resultados apresentaram fadiga no músculo flexor radial do carpo com a intensidade de $60 \%$. Porém, Hoozemans e Van Dieën (2005) verificaram menor atribuição do músculo flexor radial do carpo para mensuração da força máxima palmar, podendo ser um dos fatores para não manifestação de queda do desempenho. Outra hipótese que pode ser atribuída é de que ações isométricas exigem a compensação do sistema neuromuscular devido ao seu maior recrutamento de unidades motoras para contrabalancear a saturação de fibras que já estão em fadiga, evitando então a falha imediata do sistema (SOUZA et al., 2013).

A escala de prontidão apontou elevados níveis de fadiga, tensão e declínio nos índices de vigor. Corroborando com esses achados, Silva et al. (2018) analisam as respostas de escalas psicométricas pré e pós-treinamento resistido com restrição de fluxo sanguíneo e identificaram aumento significante da fadiga (pré: 2,6 $\pm 2,4$; pós: 7,0 $\pm 3,9 ; \mathrm{p}=0.001$; delta=169.2\%) em jogadores de basquetebol. Supõe-se que o acúmulo de metabólitos após práticas de treinamento seja um dos motivos para originar fadiga (POPE, Z. K; WILLARDSON, J. M; SCHOENFELD, B. J, 2013). Tais características apresentadas com elevada frequência podem ocasionar efeitos adversos, dentre os quais se destacam a queda de desempenho associada a fatores psicológicos e fisiológicos (NOCE et al.,2008).

Em suma, os achados da presente investigação indicam que parece haver efeito periférico importante após sessões de HIRT, mas com efeito modesto no sistema neuromuscular, o que poderia explicar a rápida recuperação na capacidade de produzir potência. Entretanto, algumas limitações devem ser consideradas para interpretação desses achados: i) a ausência de medidas diretas de fadiga periférica, como marcadores sanguíneos de dano muscular; por outro lado, para minimizar isso, os autores acreditam que os testes escolhidos apresentam indicadores suficientes para permitir essas inferências, porém, com maior validade externa; ii) a ausência de grupo controle; apesar de reconhecer a importância do grupo de comparação, os 
autores se limitaram ao objetivo central de descrever as respostas agudas e sugerem que futuras investigações proponham comparações com outros modelos de treinamento.

\section{Conclusão}

O treinamento resistido de alta intensidade (HIRT) afeta de modo agudo a potência de membros inferiores, mas não de superiores. Por outro lado, esse prejuízo parece ser suficientemente recuperado já nas primeiras $24 \mathrm{~h}$.

\section{Referências}

AMERICAN COLLEGE OF SPORTS MEDICINE position stand. Progression models in resistance training for healthy adults. Medicine and science in sports and exercise, New York, v. 41, n. 3, p. 687-708, mar. 2009.

BAPTISTA, M. T. et al. Teste de força de preensão manual: estudo da fadiga mioelétrica do flexor radial do carpo e flexor superficial dos dedos. Revista Brasileira Educação Física e Esporte, São Paulo, v. 27, n. 3, p. 345-353, abr. 2013.

BORG, Gunnar A.V. Psychophysical bases of perceived exertion. Medicine \& Science in Sports \& Exercise, v. 14, p. 377-381, 1982.

CASTRO-PIÑERO, J. et al. Assessing Muscular Strength in Youth: Usefulness of Standing Long Jump As a General Index of Muscular Fitness. Journal of Strength and Conditioning Research, p. 1810-1817, 2010.

CASTRO-PINERO, J. et al. Percentile values for muscular strength field tests in children aged 6 to 17 years: influence of weight status. Journal of strength and conditioning research, United States, p. 2295-2310, nov. 2009.

CLARSEN, B.; MYKLEBUST, G.; BAHR, R. Development and validation of a new method for the registration of overuse injuries in sports injury epidemiology: the Oslo Sports Trauma Research Centre (OSTRC) overuse injury questionnaire. British journal of sports medicine, England, v. 47, n. 8, p. 495-502, maio 2013.

CLEBIS, Naianne Kelly; RAQUEL, Maria; NATALI, Marçal. Lesões musculares provocadas por exercícios excêntricos. Muscular lesions provoked by eccentric exercise, p. 47-53, 2001. EBBELING, C. B.; CLARKSON, P. M. Exercise-induced muscle damage and adaptation. Sports medicine, New Zealand, v. 7, n. 4, p. 207-234, abr. 1989.

FETT, J. et al. Impact of physical performance and anthropometric characteristics on serve velocity in elite junior tennis players. Journal of Strength and Conditioning Research, United States, 2008.

FREITAS DE LIMA, L.; MOREIRA, O. C.; CASTRO, E. F. Efeito de quatro dias consecutivos de jogos sobre a potência muscular, estresse e recuperação percebida, em jogadores de futsal. Revista Brasileira de Educação Física e Esporte. São Paulo, v. 847, n. 2, p. 507-513, jan./mar. 2014. 
HOOZEMANS, M. J. M.; DIEEN, J. H. VAN. Prediction of handgrip forces using surface EMG of forearm muscles. Journal of electromyography and kinesiology : official journal of the International Society of Electrophysiological Kinesiology, v. 15, n. 4, p. 358-366, ago. 2005.

HUNTER, Angus M et al. Electromyographic (EMG) normalization method for cycle fatigue protocols. Medicine \& Science in Sports \& Exercise, n. 6, p. 857-861, 2001.

KRAEMER, W. J.; RATAMESS, N. A. Fundamentals of resistance training: progression and exercise prescription. Medicine and science in sports and exercise, New York, v. 36, n. 4, p. 674-688, abr. 2004.

LAN, M. F. et al. Validity of the Brunel Mood Scale for use with Malaysian athletes. Journal of Sports Science and Medicine, England, p. 131-135, mar. 2018.

LIZ, C. M. DE; ANDRADE, A. Análise qualitativa dos motivos de adesão e desistência da musculação em academias. Revista Brasileira de Ciências do Esporte, Santa Catarina, v. 38, n. 3, p. 267-274, dez. 2016.

LOPES, C. R. et al. Efeito do intervalo entre sessões de exercício de força sobre o desempenho neuromuscular. Revista Brasileira de Medicina do Esporte, São Paulo, v. 20, n. 5, p. 402-405, set./out. 2014.

MARSHALL, Paul W M et al. Acute neuromuscular and fatigue responses to the rest-pause method. Journal of Science and Medicine in Sport, Sydney, p. 153-158, 2018.

MIDDLETON, L. E.; YAFFE. K. Promising strategies for the prevention of dementia. Arch Neurol, v. 66, p. 1210-1215, 2009.

MIRANDA, H. et al. Análise de freqüência cardíaca, pressão arterial e duplo-produto em diferentes posições corporais nos exercícios resistidos. Revista Brasileira de Medicina do Esporte, São Paulo, v. 11, n. 5, p. 295-298, set./out. 2005.

MORO, T. et al. High intensity interval resistance training (HIIRT) in older adults: Effects on body composition, strength, anabolic hormones and blood lipids. Experimental Gerontology, v. 98, n. 1, p. 91-98, Aug. 2017.

NOCE, F. et al. Análise dos sintomas de overtraining durante os períodos de treinamento e recuperação: Estudo de caso de uma equipe Feminina da Superliga de voleibol 2003/2004. Revista Brasileira de Medicina do Esporte, São Paulo, v. 17, n. 6, p. 397-400, nov./dez. 2011.

PAOLI, Antonio et al. High-Intensity Interval Resistance Training (HIRT) influences resting energy expenditure and respiratory ratio in non-dieting individuals. Journal of Translational Medicine, Padova, p. 1, nov. 2012.

PFISTER, P. B. et al. Manual muscle testing and hand-held dynamometry in people with inflammatory myopathy: An intra- and interrater reliability and validity study. PLOS ONE, United States, p. 1-22, mar. 2018. 
POPE, Z. K.; WILLARDSON, J. M.; SCHOENFELD, B. J. Exercise and blood flow restriction. Journal of Strength and Conditioning Research, v. 27, n. 10, p. 2914-2926, out. 2013.

SABIDO R, PEÑARANDA M, HERNÁNDEZ-DAVÓ JL. Comparison of acute responses to four different hypertrophy-oriented resistance training Methodologies. Eur J Hum Mov, v. 37, p.109-121, 2016.

SAKUGAWA, R. L. et al. Effects of resistance training, detraining, and retraining on strength and functional capacity in elderly. Aging Clinical and Experimental Research, Germany, 2018.

SILVA, J. C. G. et al. Mood Effects of Blood Flow Restriction Resistance Exercise Among Basketball Players. Perceptual and Motor Skills, p.1-14, 2018.

SOUSA, I. C. De et al. Fadiga dos músculos extensores do punho diminui a força de preensão palmar. Revista Brasileira de Nutrição Esportiva, p. 256-262, 2013.

STONE, M.; PLISK, S.; COLLINS, D. Training principles: evaluation of modes and methods of resistance training--a coaching perspective. Sports Biomechanics, Scotland, v. 1, n,1, p. 79-103, 2002.

WEAKLEY, J. J. S. et al. The effects of traditional, superset, and tri-set resistance training structures on perceived intensity and physiological responses. European Journal of Applied Physiology, v. 117, n. 9, p. 1877-1889, 2017.

Recebido em: 18/03/2018

Revisado em: 29/08/2018

Aprovado em: 08/11/2018

Endereço para correspondência:

antenorcalandrini@gmail.com

Antenor Barbosa Calandrini Azevedo

Universidade Federal do Pará

Rua Augusto Corrêa, 01 - Guamá.

CEP 66075-110

Belém - Pará - Brasil 